\title{
FODMAPs, inflammatory bowel disease and gut microbiota: updated overview on the current evidence
}

\author{
Catarina D. Simões ${ }^{1,2}$ (1) $\cdot$ Marta Maganinho' $\cdot$ Ana S. Sousa ${ }^{1,3}$ (1)
}

Received: 22 July 2021 / Accepted: 25 November 2021

(c) The Author(s), under exclusive licence to Springer-Verlag GmbH Germany 2021

\begin{abstract}
Purpose Based on the fermentable oligosaccharides, disaccharides, monosaccharides and polyols (FODMAP) hypothesis, the low-FODMAP diet has been suggested as a potential therapeutic approach for inflammatory bowel disease (IBD) with promising results on disease management. However, this diet implies a specific broad food restriction, which potentially increases the risk of nutritional deficiencies and may aggravate gut microbiota dysbiosis of IBD patients. The aim of the present study is to review the effect of individual FODMAPs on the human gut microbiota. In addition, this narrative review provides an updated overview of the use of the low-FODMAP diet in IBD, namely the implementation, advantages, limitations, and the impact on the gut microbiota.

Methods The literature search strategy was applied to PubMed and Web of Science using relevant keywords, IBD, FODMAPs, Fructose, Lactose, Polyols, FOS, GOS, low-FODMAP diet and gut microbiota.

Results Current data suggest that the low-FODMAP diet may effectively improve clinical outcomes in the management of IBD and ensure better quality of life for IBD patients. However, there is evidence highlighting some issues of concern, particularly the adequacy of the diet and the impact on the gut microbiota. The various FODMAP types differently modulate the gut microbiota.

Conclusion IBD management should be achieved with the least possible dietary restriction to avoid detrimental consequences, particularly on nutritional adequacy and gut microbiota. Thus, it is important to individualize and monitor the nutrition intervention. Further studies are required to better characterize the relationship between diet, the gut microbiota, and IBD to support the generalization of this approach for clinical practice in IBD therapy and management.
\end{abstract}

Keywords Diet therapy $\cdot$ Inflammatory bowel diseases $\cdot$ Microbiota $\cdot$ FODMAPs $\cdot$ Low-FODMAP diet

\section{Introduction}

Inflammatory bowel disease (IBD) is a chronic relapsing intestinal inflammation, mainly represented by Crohn's disease (CD) and ulcerative colitis (UC). CD is characterized by the presence of ulcerations and/or granulomatous lesions affecting the entire bowel wall, while in UC the lesions start in the rectum and frequently extend in a continuous manner

Ana S. Sousa

asofiasousa@ufp.edu.pt

1 Faculty of Health Sciences, University Fernando Pessoa, Porto, Portugal

2 CIBIO-InBIO Research Centre in Biodiversity and Genetic Resources, University of Porto, Vairão, Portugal

3 Center for Innovative Care and Health Technology (ciTechcare), Polytechnic of Leiria, Leiria, Portugal through the colon, being usually limited to the mucosa surface [1, 2]. IBD is considered to be a disease of westernized countries [3]. Along with the rapid industrialization and lifestyle changes of modern societies, the incidence and prevalence of this pathology has been increasing through the years [3]. Between 1990 and 2017, the global prevalent cases of IBD increased 85.1\% [4]. North America and Western and Northern Europe have the highest rates and it is estimated that 1 in 198 individuals have UC and 1 in 310 individuals have CD [4]. Populations from westernized countries tend to follow hypercaloric eating patterns including ultraprocessed foods and a sedentary lifestyle [5]. This lifestyle is associated with the increased incidence of chronic diseases, specifically inflammatory diseases such as IBD, as well as diabetes, obesity and cardiovascular diseases [5].

Although the aetiology of IBD remains largely unknown, this condition is considered to be a multifactorial disease 
resulting from the contribution and interaction of several factors: genetic susceptibility, immune responses, gut microbiota and environmental factors [6,7]. Dietary pattern is a major part of the environmental triggers for the disease, particularly those diets that include a high quantity of refined carbohydrates, red meat, saturated fat, and processed foods. Diet impacts the immune system homeostasis and may contribute to intestinal inflammation through different mechanisms, including modulation of the gut microbiota [8].

The gut microbiota of a healthy adult is dominated by a small number of phyla: Firmicutes, Bacteroidetes and Actinobacteria [9-11]. In addition, lower amounts of Proteobacteria, Verrucomicrobia, Euryarchaeota, and Fusobacteria have been identified in human faecal samples [10]. The composition of the intestinal microbiota is influenced by host and environmental factors, such as diet, that continuously modulate this microbial community [12]. Although diet is deemed one of the main factors with impact on the gut microbiota, inter-individual variation may account for the majority of the compositional variation [13-15].

Homeostatic imbalance within the gut microbiota may lead to dysbiosis, i.e., an altered microbiota composition. The question of whether human gut microbiota dysbiosis is truly causative or merely a consequence of inflammation in IBD is still unclear [16]. Some studies support the hypothesis that the overall dysbiosis observed in IBD patients might be a result of the disturbed gut environment rather than the direct cause of disease [17, 18]. Conversely, other studies support the idea that gut dysbiosis is a prerequisite for inflammation rather than being driven by it [19-21]. In fact, changes in the composition of the gut microbiota do lead to microbial metabolite alterations that may have a role in IBD pathogenesis $[22,23]$. It is likely that failure to adequately regulate the microbiota composition is at the onset of many chronic diseases since a dysbiotic configuration is commonly linked to impaired epithelial barrier function and inflammation [24]. Dysbiosis of the gut microbiota may contribute to IBD pathogenesis as a result of a breakdown in the equilibrium between putative protective species and inflammatory species [25]. Protective species interact with the immune system contributing to the homeostatic mechanism while inflammatory species promote non-immunogenic inflammatory reactions, disturbing homeostasis. An abnormal immune response to the gut microbiota in genetically susceptible subjects is one of the most accepted theories behind the aetiology of IBD [26]. Most of the studies in IBD patients reported alterations in the abundance of specific bacterial taxa within the phyla Firmicutes, Bacteroidetes, Actinobacteria and Proteobacteria, with an expansion of putative inflammatory groups combined with a reduction of protective groups $[27,28]$.

Protective groups generally reduced in IBD patients include members of the phylum Firmicutes, such as the butyrate producers belonging to the genera Faecalibacterium, Roseburia, Oscillibacter and Coprococcus [19, 29]. A reduction of these genera contributes to a decrease in the production of butyrate that may predispose the intestinal mucosa to inflammation [30]. In addition, recent evidence suggests that mucosal inflammation in IBD patients may alter the capacity of the epithelium to respond to butyrate, suggesting a potential role of inflammation in decreasing butyrate uptake and metabolism [31]. Furthermore, the genus Bifidobacterium (phylum Actinobacteria) and the mucolytic bacteria Akkermansia muciniphila (phylum Verrucomicrobia) are also generally decreased in IBD patients compared to healthy subjects $[32,33]$. The presence of A. muciniphila reduces histological damage of the intestinal mucosa, and tissue mRNA expression of pro-inflammatory mediators in an animal model [34]. The expansion of putative inflammatory groups including members the family Enterobacteriaceae (phylum Proteobacteria) such as Escherichia coli, and Fusobacterium spp. (phylum Fusobacteria), has been strongly associated with IBD [35]. Moreover, the species Ruminococcus gnavus, producer of an inflammatory polysaccharide, is enriched in IBD patients [36].

Although a gut microbiota imbalance occurs in IBD patients, dysbiosis associated with CD and UC seems to be disease specific [37, 38]. Differences in the gut microbial composition have been reported between members of the same family and twins discordant for IBD [39], suggesting that dysbiosis is primarily associated with disease state rather than environmental or genetic factors [16].

Abdominal pain, bloating and modification in stool consistency and frequency are the most frequent symptoms of IBD [40]. In addition, approximately $30 \%$ of IBD patients exhibit abdominal pain associated with changes in intestinal motility [40]. IBD is associated with severe intestinal damage and requires lifelong treatment especially if onset of the disorder is early in life [1]. Moreover, the progressive increase in IBD prevalence, particularly in western countries, may increase the risk of surgery and morbidity, with higher healthcare costs [1]. Thus, appropriate, lifelong management of IBD is of utmost importance.

The current evidence on dietary strategies as primary therapy in IBD is growing and there are promising results and ongoing research. Nutrient composition of daily diets including protein, fat, carbohydrate and dietary fibre have different effects on IBD management [41]. Therefore, nutrition interventions may play an important role in the improvement of IBD symptoms and in disease remission [42]. In the past few years, several dietary interventions have been studied for potential therapeutic effects on IBD [43]. Among those interventions are the specific carbohydrate diet [44], exclusive enteral nutrition [45], gluten-free diet [46], and the anti-inflammatory diet [47]. However, these diets are either 
very restrictive and not tolerated, or there is lack of evidence as to their efficacy on IBD.

Recently, the benefits of the Mediterranean diet in the treatment and management of IBD have also been studied and the results, although scarce, seem to be promising [48]. This diet is characterized by an abundance of beneficial nutrients such as phytonutrients, monounsaturated and n-3 polyunsaturated fatty acids, fibre, and a low intake of saturated fatty acids [49]. These features have been associated with anti-inflammatory effects, particularly in CD patients [50]. Moreover, when compared with other approaches, individuals following this diet are less susceptible to nutrient deficiencies [51]. However, further research is needed on the anti-inflammatory potential of the Mediterranean diet. A promising nutritional approach directly targeting the gut microbiota was proposed by Gibson et al. [52]. This approach consists of a diet low in Fermentable Oligosaccharides, Disaccharides, Monosaccharides and Polyols (FODMAP). The use of the low-FODMAP diet in IBD has been reviewed previously $[53,54]$. This narrative review, besides discussing the implementation of the low-FODMAP diet in IBD, provides an updated overview of the effect of individual FODMAPs on the human gut microbiota. The literature search strategy was applied to PubMed and Web of Science in the last 20 years using relevant keywords, IBD, FODMAPs, Fructose, Lactose, Polyols, FOS, GOS, lowFODMAP diet and gut microbiota. No systematic assessment was performed.

\section{FODMAPs}

FODMAPs are short-chain carbohydrates with a small molecular size and a high osmotic effect that are poorly absorbed in the gastrointestinal tract. Consequently, a significant portion of the ingested FODMAPs reach the distal ileum and colon intact and are available to be used by the gut microbiota. These characteristics potentiate increasing fermentation by the gut microbiota with associated symptoms that include gas production, abdominal pain, bloating, cramping, distension and diarrhoea [40]. Excessive delivery of FODMAPs to the distal small intestine promotes bacterial overgrowth that may lead to increased intestinal permeability as a result of reduced expression of mucosal tight junctions [55, 56]. According to the FODMAP hypothesis, excessive intake of monosaccharides, disaccharides, oligosaccharides and polyols is associated with, although not proven to cause, higher susceptibility to the development of IBD and exacerbation of symptoms within IBD patients [52, 57]. The impact of the ingestion of individual FODMAPs on the human gut microbiota composition is summarized in Table 1.

\section{Monosaccharides}

Monosaccharides are the simplest form of carbohydrates that are able to traverse the intestinal epithelium by facilitated diffusion or active transport [58]. Fructose is a 6-carbon monosaccharide, mainly absorbed through carriermediated facilitative diffusion by glucose transporter 2 (GLUT2) and GLUT5 [59]. GLUT2 transports not only fructose but also glucose and galactose, whereas GLUT5 has exclusive affinity for fructose [59]. The ingestion of high fructose levels may compromise fructose absorptive capacity leading to malabsorption. In addition, low expression of GLUT5 in infants potentially explain fructose malabsorption in young children [59]. Common contributors to fructose intake include fresh fruits, fruit-derived products, and products containing high-fructose sweeteners such as corn syrup.

The impact of a high-fructose diet on the human gut microbiota is still largely unknown. A recent study investigated the influence of two consecutive short-term fructoserich diets (100 g/day) on the gut microbiota composition of female subjects: first a high-fructose diet rich in fruits and vegetables (fruit diet) followed by a high fructose syrup diet, both implemented after a low-fructose phase [60]. At the genus level, specific deviations were observed including an increase in Faecalibacterium and Anaerostipes and a reduction in Parabacteroides and Barnesiella after the fruit-rich diet. Conversely, the high-fructose syrup diet induced a decrease of Ruminococcus, Faecalibacterium and Erysipelatoclostridium whereas the abundance of Barnesiella increased. Although the amount of fructose ingested in both diets was similar, the fruit diet contained a higher fibre content than the high fructose syrup diet. Therefore, the alterations in gut microbiota composition observed may reflect the different intake of fibre rather than changes in response to the high-fructose consumption [60]. The genera Anaerostipes, Coprococcus, Ruminococcus and Erysipelatoclostridium are butyrate-producing bacteria of the phylum Firmicutes. Butyrate is a short chain fatty acid (SCFA) that is absorbed by the intestinal mucosa where it is the main energy source for colonocytes and is involved in the regulation of immunity and inflammation [61]. In addition, butyrate has anti-inflammatory effects, it modulates cytokine production, induces expansion of regulatory $\mathrm{T}$ cells, and positively modulates intestinal homeostasis [61]. On the other hand, Barnesiella is implicated in the induction of the proinflammatory IFN $\gamma$ response [62]. Therefore, while a high fructose fruit diet promotes the growth of beneficial butyrate-producing bacteria, a high-fructose syrup diet reduces their abundance, promotes proinflammatory cytokine production and consequently compromises gut homeostasis [60]. 
Table 1 Effect of individual FODMAPs on human gut microbiota composition

\begin{tabular}{|c|c|c|c|}
\hline FODMAP & Study description & Gut microbiota effect ${ }^{\mathrm{a}}$ & References \\
\hline \multirow[t]{2}{*}{ Fructose } & $\begin{array}{l}\text { Intervention study, } n=12,1 \text {-week low-fructose diet }(10 \mathrm{~g} / \\
\text { day), 1-week Fruits and vegetables diet ( } 100 \mathrm{~g} / \text { day })\end{array}$ & $\begin{array}{l}\uparrow \text { Faecalibacterium, } \uparrow \text { Anaerostipes, } \downarrow \text { Barnesiella, } \downarrow \text { Para- } \\
\text { bacteroides }\end{array}$ & {$[60]$} \\
\hline & $\begin{array}{l}\text { Intervention study, } n=12,1 \text {-week low-fructose diet ( } 10 \mathrm{~g} / \\
\text { day), 1-week High-fructose syrup diet ( } 100 \mathrm{~g} / \text { day })\end{array}$ & $\begin{array}{l}\downarrow \text { Ruminococcus, } \downarrow \text { Faecalibacterium, } \downarrow \text { Erysipelato- } \\
\quad \text { clostridium, } \uparrow \text { Barnesiella }\end{array}$ & {$[60]$} \\
\hline \multirow[t]{2}{*}{ Lactose } & $\begin{array}{l}\text { Cross-sectional study; } n=1068 \text {, Assessment of dairy } \\
\text { products intake through a 7-day Food frequency ques- } \\
\text { tionnaire }\end{array}$ & $\uparrow$ Bifidobacterium & {$[64]$} \\
\hline & $\begin{array}{l}\text { Cross-sectional study; } \mathrm{n}=959 \\
\text { Assessment of dairy products intake through a Brief-type } \\
\text { diet history questionnaire }\end{array}$ & $\uparrow$ Bifidobacterium & {$[65]$} \\
\hline \multirow[t]{3}{*}{ FOS } & $\begin{array}{l}\text { Randomized clinical trial; } n=36,14 \text {-day supplementation: } \\
16 \text { g/day FOS }\end{array}$ & $\uparrow$ Bifidobacterium & [71] \\
\hline & $\begin{array}{l}\text { Randomized controlled trial; } n=80,75 \text {-day supplementa- } \\
\text { tion: } 2.5,5 \text { and } 10 \mathrm{~g} / \text { day FOS or placebo (maltodextrin) }\end{array}$ & $\uparrow$ Bifidobacterium, $\uparrow$ Lactobacillus, $\uparrow$ Faecalibacterium & {$[72]$} \\
\hline & $\begin{array}{l}\text { Randomized controlled trial; } n=25,6 \text {-week supplementa- } \\
\text { tion: } 16 \mathrm{~g} / \text { day inulin-type fructan or placebo (maltodex- } \\
\text { trin) }\end{array}$ & $\uparrow$ Bifidobacterium & [73] \\
\hline \multirow[t]{2}{*}{ GOS } & $\begin{array}{l}\text { Randomized controlled trial; } n=151,3 \text {-week supplemen- } \\
\text { tation: } 5 \mathrm{~g} / \text { day GOS }\end{array}$ & $\uparrow$ Bifidobacterium, $\uparrow$ Lactobacillus & [70] \\
\hline & $\begin{array}{l}\text { Randomized clinical trial; } n=36,14 \text {-day supplementation: } \\
16 \text { g/day GOS }\end{array}$ & $\uparrow$ Bifidobacterium & [71] \\
\hline Xylitol & In vitro human fecal culture from five healthy donors & $\uparrow$ Anaerostipes & {$[82]$} \\
\hline Lactitol & $\begin{array}{l}\text { Randomized clinical trial, } n=75,7 \text {-day intake of a choco- } \\
\text { late bar containing } 10 \mathrm{~g} \text { sweetener in the ratios 10:0, 5:5 } \\
\text { or 0:10 sucrose:lactitol }\end{array}$ & $\uparrow$ Bifidobacterium & {$[88]$} \\
\hline Isomalt & $\begin{array}{l}\text { Double-blind controlled trial, } n=19,2 \times 4 \text {-week controlled } \\
\text { basal diet enriched with either } 30 \mathrm{~g} \text { isomalt or } 30 \mathrm{~g} \\
\text { sucrose daily }\end{array}$ & $\uparrow$ Bifidobacterium & {$[86]$} \\
\hline
\end{tabular}

${ }^{\mathrm{a}}$ Main genus affected

\section{Disaccharides}

Disaccharides are composed of two monosaccharide units linked by glycosidic bonds. Lactose is a disaccharide composed of glucose and galactose residues. While dairy products are the main dietary sources of lactose, it is also added to commercial foods such cakes and breads. Lactose is hydrolyzed at the intestinal brush border by the enzyme lactase. However, humans present lactose malabsorption due to a lower level of or inability to produce lactase, or inadequate enzyme activity [63]. This impairment causes an increase of undigested lactose in the colon, where it is fermented by the gut microbiota with production of SCFAs and gases [63], leading to abdominal discomfort, bloating, burping, flatulence, and nausea. These symptoms may result from increased abundance of Bifidobacterium spp. in the gut rather than being a direct effect of lactose intake $[64,65]$.

\section{Oligosaccharides}

Oligosaccharides are a class of carbohydrates containing up to 10 monosaccharide units such as fructooligosaccharides
(FOS) and galactooligosaccharides (GOS). Humans are unable to digest these oligosaccharides since they lack the enzymes to break them down [66]. FOS are fructans, i.e. fructose polymers, composed of a small number of fructose units linked by $(2 \rightarrow 1)-\beta$-glycosidic bonds with a single D-glucosyl unit at the non-reducing end [67]. Dietary fructans are mostly FOS and food sources include garlic, rye, barley, pistachio, peach, watermelon, artichoke, leek, wheat and onion [52, 66]. Commercial fructans are increasingly added to processed foods due to their sensory and textural properties and potential health benefits such as prebiotic effect and low-energy content. GOS are composed of galactose monomers with a terminal glucose unit at the reducing end linked by $\beta$-glycosidic bonds. The dietary forms of GOS are raffinose and stachyose that are not hydrolysed in the gastrointestinal tract due to the lack of the enzyme $\beta$-galactosidase [68]. Common food sources include lentils, beans, cabbage, chickpeas, Brussels sprouts, chicory, onion, some grains, nuts and seeds.

FOS and GOS are dietary fibres that resist the hydrolytic actions of intestinal enzymes. These oligosaccharides are considered prebiotics, i.e., a "non-digestible food ingredient 
that beneficially affects the host by selectively stimulating the growth and/or activity of one or a limited number of bacteria in the colon, thereby improving the host's health" [69]. FOS and GOS confer growth-promoting effects on beneficial microbes such as Bifidobacterium spp. and Lactobacillus spp. [68, 70-73]. The members of these genera mainly produce the SCFA acetate and propionate, and also lactate, which downregulate the proinflammatory response of intestinal epithelial cells and inhibit the growth of potentially pathogenic organisms [74], contributing to the maintenance of colonic homeostasis. However, recent studies have reported that these prebiotic carbohydrates may also be metabolized by the extraintestinal avian-pathogenic strain Escherichia coli BEN2908 [75], and by the human commensal E. coli CCFM 8331 [76], contributing to their intestinal colonization which may constitute an increased risk to human health.

\section{Polyols}

Polyols are a specific group of sugar alcohols that are formed via the catalytic hydrogenation of carbohydrates, and include substances such as sorbitol, mannitol, maltitol, lactitol and xylitol. Only approximately one-third of polyols that are consumed in the human diet are absorbed in the small intestine via passive diffusion. The rate of absorption varies depending on multiple factors such as the molecular size of the polyol, the amount ingested, the pore size in the distal small intestine and the presence of gastrointestinal disorders [77]. Polyol malabsorption varies according to the substrate, is generally dose-dependent, and increases when polyols are ingested in combination with other carbohydrates. An exception is erythritol, which is relatively well absorbed compared with the other polyols. Unabsorbed polyols that reach the colon are available to be fermented by the gut microbiota with production of gas (hydrogen, carbon dioxide, and methane) and other end products of fermentation [78]. Therefore, polyols may induce dose-dependent gastrointestinal symptoms of abdominal pain, flatulence, bloating and osmotic diarrhoea [79]. Polyols such as sorbitol and mannitol occur naturally in fruits and vegetables such as apple, pear, apricot, cherry, nectarine, peach, plum, watermelon, mushroom, cauliflower, and also honey [78, 80, 81]. Polyols also include artificial sweeteners (e.g., xylitol, lactitol and isomalt) frequently used by the food industry as sugar substitutes to produce sugar-free and low-calorie content products as well as to fulfil technological functions, such as emulsifiers, stabilizers and texturizers [79].

The effects of polyols on the human gut microbiota have been studied for the artificial sweeteners xylitol, isomalt, and lactitol. To our knowledge there are no data available about the effect of naturally occurring sorbitol and mannitol on gut microbiota.
Xylitol supplementation ( $0.5 \%)$ of faecal cultures in vitro increased the abundance of the species within the Anaerostipes genus, and bacteria within the Bacteroides fragilis group [82]. B. fragilis has been associated with the increase of Interleukin 10 (IL-10)-producing regulatory T cells, which limit the proinflammatory mechanisms and consequently contribute to reduction of intestinal inflammation [83]. In addition, Anaerostipes has been identified as a potential biomarker for Irritable Bowel Syndrome (IBS) and IBD since the abundance of the genus is reduced in patients with these gastrointestinal disorders, more pronouncedly in IBD [84]. In addition, xylitol stimulated the formation of butyrate, in higher amounts than supplementation with the same amount of FOS and GOS [82, 85].

The effect of a diet enriched with isomalt on the gut microbiota of healthy individuals has been assessed through a double-blind placebo-controlled study and compared to a diet enriched with the same amount of sucrose [86]. The isomalt diet resulted in a statistically significant increase of Bifidobacteria when compared to the sucrose diet, whereas the abundance of Lactobacillus, Bacteroides, Enterococcus, and E. coli did not differ between the two diets. According to the study, isomalt is a potential prebiotic that may contribute to a healthy gut environment by stimulating the growth of beneficial bacteria [86].

Lactitol is a sweet-tasting sugar alcohol derived from lactose [87]. The effect of a low dose lactitol on the faecal microbiota has also been studied in a randomized, longitudinal, double-blind study with 75 healthy volunteers who consumed $25 \mathrm{~g}$ tablets of milk chocolate containing $10 \mathrm{~g}$ sucrose and lactitol in ratios of 10:0, 5:5, or 0:10 daily for 7 days [88]. While the faecal microbiota of the individuals consuming 0 and $5 \mathrm{~g}$ of lactitol remained relatively stable, the consumption of $10 \mathrm{~g}$ of lactitol resulted in a significant increase in the abundance of Bifidobacterium spp., increased production of propionate and butyrate and a significant decline in faecal $\mathrm{pH}$ that may contribute to the reduction of pathogenic bacteria such as members of the family Enterobacteriaceae [88]. Although Bifidobacteria are not butyrate producers, the production of acetate may stimulate the growth of beneficial butyrogenic species. Therefore, low doses of lactitol may function as a prebiotic and be beneficial in inducing a healthy gut microbiota.

Generally, polyol malabsorption is dose-dependent in healthy individuals. The simultaneous ingestion of different polyols tends to aggravate the malabsorption [78]. However, studies on polyol malabsorption and their effect on gut microbiota composition in patients with gastrointestinal disorders are limited, particularly in IBD. Polyols may induce dose-dependent symptoms of flatulence, abdominal discomfort, intestinal dysmotility and laxative effects when consumed by both healthy volunteers and patients with IBS [78]. Further research is needed to better understand 
the effects of specific polyols on gastrointestinal function and microbiota composition in both healthy individuals and those with gastrointestinal disorders such as IBD.

\section{Low-FODMAP-Diet in IBD}

Based on the FODMAP hypothesis, the low-FODMAP diet has been suggested as a potential therapeutic approach for IBD patients, mainly due to the promising results on disease management, especially on controlling functional intestinal symptoms $[89,90]$.

The self-reported perception of the influence of specific foods on IBD symptoms was evaluated by Cohen et al. [91] in a cohort study of 6,768 IBD patients. The study assessed dietary consumption patterns and identified foods that patients more often associated with the improvement or worsening of their IBD symptoms. The reported dietary patterns differed by disease type and disease activity [91]. IBD patients restrict their dietary patterns due to the presence of active symptoms or the fear of exacerbation [90]. Given patients' self-reported perception of the influence of specific foods on IBD and considering the effects of FODMAPs on disease worsening, the role of diet as a triggering factor for intestinal functional symptoms becomes clear [66, 92, 93]. The low-FODMAP diet has been applied in patients with IBD mainly due to the success of its implementation in patients with IBS, with great efficacy in reducing intestinal functional symptoms [94].

The implementation of a low-FODMAP diet can follow two different approaches: top-down or bottom-up [94]. The top-down approach consists of restricting all or almost all foods rich in FODMAPs, for a period of 4-8 weeks. The bottom-up approach, on the other hand, entails the reduction of specific FODMAPs or foods that are very rich in FODMAPs for a certain period of time, and subsequently restricting other foods if deemed necessary [94]. However, most of the available guidelines mention that the elimination of FODMAPs should be based on a global restriction [95]. There are few studies reporting results on the implementation of the low-FODMAP diet in IBD patients.

Gearry et al. [89] implemented the low-FODMAP diet in a pilot study with 72 IBD patients. Most of the symptoms improved significantly after following this approach.

Prince et al. [90], in a case note review of electronic medical records of 88 IBD patients who had been on a low-FODMAP diet, reported that at follow-up there was an increase in the proportion of patients with satisfactory relief of functional bowel symptoms along with normal stool frequency and consistency when compared to patients at baseline.

Through a randomized controlled clinical trial, Pedersen et al. [96] studied 89 patients with remission IBD or with mild to moderate disease activity with coexistence of
IBS-like symptoms. In this clinical trial, two distinct groups were studied: the low-FODMAP diet (intervention group) and normal diet (control group) for 6 weeks. At the end of the intervention period, there was a reduction in the severity of symptoms in the low-FODMAP diet group, indicating that patients following this diet are more likely to experience reduction of IBS-like symptoms than patients on a regular $\operatorname{diet}[96]$.

Furthermore, Bodini et al. [97] reported a significant decrease of disease activity in CD patients during a sixweek low-FODMAP diet implemented in 55 IBD patients in remission or with mild disease activity. Moreover, the median value of faecal calprotectin, a sensitive marker for inflammation in the gastrointestinal tract, decreased significantly in the follow-up of patients on the low-FODMAP diet when compared with the values obtained by those following a standard diet [97].

Maagaard et al. [98] conducted a retrospective crosssectional study with patients with IBS $(n=131)$ and IBD $(n=49)$ treated with a low-FODMAP diet, to assess longterm adherence and the effect on the course of the disease. Most of the patients reported total or partial efficacy of the low-FODMAP diet with great improvement to bloating and abdominal pain [98]. After intervention with the low-FODMAP diet, the number of patients with chronic continuous disease progression decreased while the number of patients with mild and indolent disease progression increased. Patients with mild and indolent disease progression improved quality of life and presented a normal stool pattern [98]. In addition, 24\% of patients with IBD became asymptomatic after following the low-FODMAP diet [98]. Higher patient compliance was associated with a longer duration of dietary treatment in this study. However, only half of the patients followed the recommendations of the low-FODMAP diet continuously, while the others adopted the diet according to the severity of the symptoms [98]. The change of IBD patients' eating patterns according to the activity of the disease is in line with the results mentioned above.

The success of the low-FODMAP diet is associated with patients' perception of the ongoing effectiveness that the diet has in the management of symptoms [99]. Nevertheless, the effectiveness of the diet depends on the continuous adherence of patients and commitment to the diet is critical to achieving a better outcome $[89,98]$.

It is worth mentioning that the consumption of foods rich in FODMAPs is associated with aggravated gastrointestinal symptoms in patients with IBD [66, 92]. However, these FODMAPs also have a number of physiological effects that can play a beneficial role on immune function modulation, improvement of calcium absorption and stool bulk, reduction of serum cholesterol and triglycerides levels, and prebiotic effect $[92,100]$. This apparent contradiction highlights 
the importance of a personalized nutritional intervention and the need for constant monitoring. Moreover, despite the unequivocal benefits of a low-FODMAP diet in relieving the symptoms of patients with IBD, it is important to consider the possible adverse effects. There is evidence highlighting a number of issues of concern, particularly the adequacy of the diet and the impact on the gut microbiota [40, 101, 102] during the exclusion phase. However, it is worth noting that the exclusion phase should be applied for a short period of time. The main goal should always be to achieve the greatest symptom control with the least dietary restriction.

\section{Effects of a low-FODMAP diet on IBD patients}

\section{Dietary adequacy}

The major problem is the restrictive nature of the low-FODMAP diet that limits the intake of several nutrients. In the exclusion phase, foods such as fruit, vegetables, legumes, grains and grain-based products, important sources of fibre, folate, and vitamin $\mathrm{C}$, are eliminated. Calcium intake may also be deficient due to the restriction of lactose-containing dairy products. In addition, this diet potentially worsens nutritional deficiencies in a population already at high risk of malnutrition [1, 40, 92, 101]. IBD patients are particularly susceptible to zinc and vitamin D deficiencies [1, 103]. There is a high probability of vitamin D deficiency in IBD patients since vitamin D receptor polymorphisms have already been identified as a genetic factor in these patients [103]. Furthermore, due to the essential role of vitamin D in the normal functioning of the immune system, particularly in the gastrointestinal tract [1], low levels of vitamin D may be associated with dysbiosis and increased IBD-related hospitalization [104, 105]. Moreover, zinc deficiency has been linked with excessive loss of gastrointestinal secretions during chronic diarrhoea and drainage of fistulas [103]. Given that zinc is an essential enzyme cofactor for wound healing, cell immunity and growth, low levels of this micronutrient are also associated with increased hospitalization, surgery and other complications [106]. In the exclusion phase of the low-FODMAP diet, the elimination of lactose-containing dairy products and the subsequent deficient calcium intake may impair vitamin D intestinal absorption which can possibly aggravate vitamin D deficiency in these patients [107]. In addition, the elimination of dietary zinc sources, such as legumes, grains and grain-based products, may also aggravate zinc deficiency [108].

Maagaard et al. reported that $29 \%$ of patients experienced weight loss during the low-FODMAP diet, although the real impact of this reduction on the impairment of the patients' nutritional status remains unknown [98]. In addition, a randomized controlled trial carried out by Cox et al. [109] revealed that patients with IBD during the low-FODMAP diet have a significantly lower intake of energy, protein, fat, sugars, calcium, phosphorus and iodine when compared to those on a sham diet. Furthermore, the low-FODMAP diet appears to frequently affect the fibre content ingested [110], which is why constipation is reported to be the symptom that least improves with the low-FODMAP diet $[89,110]$. This seems to be explained by the restriction of the substrates responsible for supplying fluids to the intestine [89].

Through interactions with the intestinal microbiota, fibre helps in maintaining the function of the intestinal barrier, preserving the inner mucosal layer and acting as the first line of defence against mucosal pathogens [111]. Thus, a reduction in fibre intake leads to a decrease in the production of SCFA, which are known to have the capacity to improve intestinal inflammation, as well as affect the composition of the intestinal microbiota [112]. Such changes are associated with the thinning of the inner mucus layer and, consequently, with the increased proximity of bacteria to the intestinal epithelium [112].

\section{The low-FODMAP diet and gut microbiota}

Data on the effect of the low-FODMAP diet on the gut microbiota and inflammatory markers or disease activity are scarce. Halmos et al. investigated the effects of two controlled diets, typical vs low-FODMAP diet, on faecal microbiota and other biomarkers of colonic health in patients with quiescent CD [113]. The low-FODMAP diet reduced the abundance of specific intestinal bacterial groups, particularly A. muciniphila and bacteria belonging to the Clostridium cluster XIVa that includes the acetate and lactate-converting butyrate producers Roseburia intestinalis and Eubacterium rectale, which are important for the health of the mucosaassociated microbiota [113]. Conversely, the low-FODMAP diet increased the relative abundance of the mucolytic Ruminococcus torques [113], which may lead to excessive degradation of mucus and bacterial invasion [114]. Although both diets had no effect on calprotectin levels, gastrointestinal symptoms were significantly less severe in patients on the low-FODMAP diet.

Cox et al. investigated the effect of a four-week lowFODMAP diet on the faecal microbiota composition of quiescent IBD patients [109]. The diet involved the restriction of dietary fructans, GOS, lactose, fructose in excess of glucose, and polyols, including sorbitol and mannitol. A significant decrease in the abundance of Bifidobacteria, in particular Bifidobacterium longum and Bifidobacterium adolescentis, and $F$. prausnitzii was reported following the low-FODMAP diet, most likely due to changes in the available fermentable substrates in the colon [109]. The decrease in the concentration of these bacteria is of particular concern given their immunoregulatory effects. $F$. prausnitzii exhibit protective effects via inflammatory cytokine regulation or, 
alternatively, stimulation of IL-10 production [115]. However, no detrimental effects of a low-FODMAP diet on the inflammatory marker faecal calprotectin were observed in the study, possibly because other bacteria, such as $R$. intestinalis and Lactobacillus spp., also exert immune-modulatory effects and were not altered by the diet [109]. Moreover, much of the evidence of the immune-regulatory effects of $F$. prausnitzii relate to strain A2-165 that did not differ between the sham diet and the low-FODMAP diet groups [109].

\section{Discussion}

Nutritional intervention has gained prominence in the treatment of IBD since food is among the main triggers of functional intestinal symptoms in these patients $[1,93]$.

After the presentation of the FODMAP hypothesis [52], several studies have been conducted in order to demonstrate the effectiveness of a low-FODMAP diet in the management of functional intestinal symptoms [92, 101]. Furthermore, the use of a low-FODMAP diet in the management of IBD can modulate the course of the disease and increase remission periods [98]. Thus, higher adherence to the diet is associated with better outcomes and better quality of life [96-99]. However, despite the positive impact of low-FODMAP diet on symptom relief, it is essential to consider the adverse effects and concerning issues associated with the implementation of this diet [40, 94, 103].

The intake of individual types of FODMAPs has an impact on gut microbiota composition in non-IDB subjects $[65,71,72,88]$. In addition, the food source of the individual FODMAPs, e.g., fructose from fruits and vegetables vs fructose from high-fructose syrup, differently modulate the gut microbiota of the same individual [60]. The different types of FODMAPs tend to promote the growth of gut beneficial bacteria. Therefore, a global restriction of food sources containing FODMAPs may have a pronounced detrimental effect on the gut microbiota and epithelial homeostasis. In fact, the low-FODMAP diet implies a drastic food restriction for a certain period, which naturally may lead to nutritional deficiencies and increased risk of malnutrition [103, 109]. Fibre content is one of the food components that is affected by the diet, which may consequently aggravate constipation in IBD patients $[89,110]$. Moreover, fibre restriction affects the normal functioning of the intestinal barrier, gut microbiota composition and the production of SCFA [111, 112]. Since IBD patients present a dysbiotic gut microbiota profile, the impact of a low-FODMAP diet on the gut microbiota calls for special attention, since it leads to a reduction of specific bacteria such as A. muciniphila and the genera Bifidobacterium and Faecalibacterium [109]. These beneficial bacteria have immunoregulatory effects and contribute to the immunological modulation through the increase of intestinal specific immunoglobulins and immuno-regulatory interleukins, as well as a reduction in pro-inflammatory interleukins [68]. Although restriction of FODMAPs in the diet is generally based on a global restriction, a personalized diet based on the restriction of individual types of FODMAPs, and based on their food sources, should be considered to attenuate the adverse effects including the microbiota dysbiosis in IBD patients.

Dietary restriction during implementation of the lowFODMAP diet may affect the presence in the colon of other dietary components that co-exist in many foods, e.g., slow fermentable or non-fermentable carbohydrates such as nonstarch polysaccharides. The reduction of these carbohydrates will likely exhaust substrates for carbohydrate fermentation in the proximal colon, promoting protein fermentation in the distal colon [116]. Whereas carbohydrate fermentation leads to perceived health-promoting metabolites, anaerobic degradation of proteins yields toxic metabolites, e.g., sulphurcontaining compounds such as ammonia, as well as phenolic and indolic compounds [117]. Dietary planning during the low-FODMAP exclusion phase should consider the delivery of slow fermentable carbohydrates to the colon, and the amount of protein and the type of amino acids consumed so as to modulate bacterial activity away from protein fermentation, therefore minimizing its harmful effects.

Besides the health implications of the restrictive nature of a low-FODMAP diet in the exclusion phase, there is also a risk of developing eating disorders [118]. Individuals with gastrointestinal disorders, e.g., IBD, following restrictive diets may be at increased risk for dysfunctional eating behaviours. Moreover, gastrointestinal symptoms in IBD patients can cause food aversions that may lead to food anxiety and, consequently, affect psychological well-being [118].

Most of the studies reporting benefits and adverse effects of the low-FODMAP diet present some limitations such as the small sample size which in turn can impair the relevance of the results, the design, and the period of diet application. Moreover, there are only a few studies showing data on the impact of the low-FODMAP diet on inflammatory markers or disease activity $[96,97,109]$. There is also lack of information about the adequacy of the low-FODMAP diet and its safety for IBD patients in long-term use.

Nutritional interventions play an important role on the management of IBD symptoms and in extending remission of the disease. The incidence of IBD is increasing, as is the number of patients on the low-FODMAP diet. However, despite all the documented benefits of the low-FODMAP diet, information on the consequences of long-term application is still scarce. Thus, ongoing clinical and nutritional monitoring is necessary to ensure that the low-FODMAP diet is adequately implemented and to prevent adverse effects. 
In conclusion, the low-FODMAP diet may effectively improve clinical outcomes in the management of IBD and ensure better quality of life for IBD patients.

\section{Implication and future perspectives}

According to the current evidence, a personalized intervention and frequent monitoring of IBD patients on the lowFODMAP diet is of utmost importance in clinical practice. Moreover, during the exclusion phase of the low-FODMAP diet, adequate amounts of slow fermentable carbohydrates, protein, and the type of amino acids should also be taken into consideration to minimize the potentially harmful effects of protein fermentation. With regard to the implementation of the diet, disease management should be achieved with the least possible dietary restriction to avoid further detrimental consequences, particularly on nutritional adequacy and gut microbiota. Further studies are required to better characterize the relationship between diet, gut microbiota, and IBD to support the generalization of this approach for clinical practice in IBD therapy and management.

Acknowledgements The authors acknowledge Dr Allison Byrne for the English revision of the manuscript.

Author contributions Conceptualization: CS, MM, ASS; original draft preparation: CS, MM, ASS; review and editing: CS, MM, ASS.

Funding Not applicable.

Availability of data and materials Not applicable.

Code availability Not applicable.

\section{Declarations}

Conflict of interest The authors declare no conflict of interest.

\section{References}

1. Ruemmele FM (2016) Role of diet in inflammatory bowel disease. Ann Nutr Metab 68(Suppl 1):33-41. https://doi.org/10. $1159 / 000445392$

2. Ordás I, Eckmann L, Talamini M et al (2012) Ulcerative colitis. Lancet 380:1606-1619. https://doi.org/10.1016/S0140-6736(12) 60150-0

3. Ng SC, Shi HY, Hamidi N et al (2018) Worldwide incidence and prevalence of inflammatory bowel disease in the 21 st century: a systematic review of population-based studies. Lancet 390:2769-2778. https://doi.org/10.1016/s0140-6736(17)32448OPMID-29050646

4. Alatab S, Sepanlou SG, Ikuta K et al (2020) The global, regional, and national burden of inflammatory bowel disease in 195 countries and territories, 1990-2017: a systematic analysis for the Global Burden of Disease Study 2017. Lancet
Gastroenterol Hepatol 5:17-30. https://doi.org/10.1016/S24681253(19)30333-4

5. Bernstein CN, Shanahan F (2008) Disorders of a modern lifestyle: reconciling the epidemiology of inflammatory bowel diseases. Gut 57:1185-1191. https://doi.org/10.1136/gut.2007. 122143

6. Annese V (2020) Genetics and epigenetics of IBD. Pharmacol Res 159:104892. https://doi.org/10.1016/j.phrs.2020.10489 2PMID-32464322

7. Turpin W, Goethel A, Bedrani L, Kenneth Croitoru MDCM (2018) Determinants of IBD heritability: genes, bugs, and more. Inflamm Bowel Dis 24:1133-1148. https://doi.org/10. 1093/ibd/izy085PMID-29701818

8. Kau AL, Ahern PP, Griffin NW et al (2011) Human nutrition, the gut microbiome and the immune system. Nature 474:327336. https://doi.org/10.1038/nature10213;10.1038/nature10213

9. Eckburg PB, Bik EM, Bernstein CN et al (2005) Diversity of the human intestinal microbial flora. Science 308:1635-1638. https://doi.org/10.1126/science.1110591

10. Arumugam M, Raes J, Pelletier E et al (2011) Enterotypes of the human gut microbiome. Nature 473:174-180. https://doi. org/10.1038/nature09944

11. Tap J, Mondot S, Levenez F et al (2009) Towards the human intestinal microbiota phylogenetic core. Environ Microbiol 11:2574-2584. https://doi.org/10.1111/j.1462-2920.2009. 01982.x

12. Spor A, Koren O, Ley R (2011) Unravelling the effects of the environment and host genotype on the gut microbiome. Nat Rev Microbiol 9:279-290. https://doi.org/10.1038/nrmic ro2540

13. Salonen A, Lahti L, Salojärvi J et al (2014) Impact of diet and individual variation on intestinal microbiota composition and fermentation products in obese men. ISME J. https://doi.org/10. 1038/ismej.2014.63

14. Simões CD, Maukonen J, Kaprio J et al (2013) Habitual dietary intake is associated with stool microbiota composition in monozygotic twins. J Nutr 143:417-423. https://doi.org/10.3945/ jn. 112.166322

15. Jones RB, Zhu X, Moan E et al (2018) Inter-niche and inter-individual variation in gut microbial community assessment using stool, rectal swab, and mucosal samples. Sci Rep 8:4139. https:// doi.org/10.1038/s41598-018-22408-4

16. Ni J, Wu GD, Albenberg L, Tomov VT (2017) Gut microbiota and IBD: causation or correlation? Nat Rev Gastroenterol Hepatol 14:573-584. https://doi.org/10.1038/nrgastro.2017.88

17. Tang MS, Poles J, Leung JM et al (2015) Inferred metagenomic comparison of mucosal and fecal microbiota from individuals undergoing routine screening colonoscopy reveals similar differences observed during active inflammation. Gut Microbes 6:48-56. https://doi.org/10.1080/19490976.2014.1000080

18. Hansen TH, Gøbel RJ, Hansen T, Pedersen O (2015) The gut microbiome in cardio-metabolic health. Genome Med 7:33 https://doi.org/10.1186/s13073-015-0157-z

19. Forbes JD, Van Domselaar G, Bernstein CN (2016) Microbiome survey of the inflamed and noninflamed gut at different compartments within the gastrointestinal tract of inflammatory bowel disease patients. Inflamm Bowel Dis 22:817-825. https://doi.org/ 10.1097/MIB.0000000000000684

20. Dharmani P, Strauss J, Ambrose C et al (2011) Fusobacterium nucleatum infection of colonic cells stimulates MUC2 mucin and tumor necrosis factor alpha. Infect Immun 79:2597-2607. https:// doi.org/10.1128/IAI.05118-11

21. Liu H, Hong XL, Sun TT et al (2020) Fusobacterium nucleatum exacerbates colitis by damaging epithelial barriers and inducing aberrant inflammation. J Dig Dis 21:385-398. https://doi.org/10. 1111/1751-2980.12909 
22. Parada Venegas D, De la Fuente MK, Landskron G et al (2019) Short chain fatty acids (SCFAs)-mediated gut epithelial and immune regulation and its relevance for inflammatory bowel diseases. Front Immunol 10:277

23. Simrén M, Barbara G, Flint HJ et al (2013) Intestinal microbiota in functional bowel disorders: a Rome foundation report. Gut 62:159-176. https://doi.org/10.1136/gutjnl-2012-302167

24. Bäckhed F, Fraser CM, Ringel Y et al (2012) Defining a healthy human gut microbiome: current concepts, future directions, and clinical applications. Cell Host Microbe 12:611-622. https://doi. org/10.1016/j.chom.2012.10.012

25. Sartor RB, Wu GD (2017) Roles for intestinal bacteria, viruses, and fungi in pathogenesis of inflammatory bowel diseases and therapeutic approaches. Gastroenterology 152:327-339.e4. https://doi.org/10.1053/j.gastro.2016.10.012

26. Strober W, Fuss I, Mannon P (2007) The fundamental basis of inflammatory bowel disease. J Clin Invest 117:514-521. https:// doi.org/10.1172/JCI30587

27. Alam MT, Amos GCA, Murphy ARJ et al (2020) Microbial imbalance in inflammatory bowel disease patients at different taxonomic levels. Gut Pathog 12:1. https://doi.org/10.1186/ s13099-019-0341-6

28. Pittayanon R, Lau JT, Leontiadis GI et al (2020) Differences in gut microbiota in patients with vs without inflammatory bowel diseases: a systematic review. Gastroenterology 158:930-946.e1. https://doi.org/10.1053/j.gastro.2019.11.294

29. Machiels K, Joossens M, Sabino J et al (2014) A decrease of the butyrate-producing species Roseburia hominis and Faecalibacterium prausnitzii defines dysbiosis in patients with ulcerative colitis. Gut 63:1275-1283. https://doi.org/10.1136/ gutjnl-2013-304833

30. Magnusson MK, Isaksson S, Öhman L (2020) The anti-inflammatory immune regulation induced by butyrate is impaired in inflamed intestinal mucosa from patients with ulcerative colitis. Inflammation 43:507-517. https://doi.org/10.1007/ s10753-019-01133-8

31. Ferrer-Picón E, Dotti I, Corraliza AM et al (2020) Intestinal inflammation modulates the epithelial response to butyrate in patients with inflammatory bowel disease. Inflamm Bowel Dis 26:43-55. https://doi.org/10.1093/ibd/izz119

32. Png CW, Lindén SK, Gilshenan KS et al (2010) Mucolytic bacteria with increased prevalence in IBD mucosa augment in vitro utilization of mucin by other bacteria. Am J Gastroenterol 105:2420-2428. https://doi.org/10.1038/ajg.2010.281

33. James SL, Christophersen CT, Bird AR et al (2015) Abnormal fibre usage in UC in remission. Gut 64:562-570. https://doi.org/ 10.1136/gutjnl-2014-307198

34. Gobert AP, Sagrestani G, Delmas E et al (2016) The human intestinal microbiota of constipated-predominant irritable bowel syndrome patients exhibits anti-inflammatory properties. Sci Rep 6:39399. https://doi.org/10.1038/srep39399

35. Nagao-Kitamoto H, Kamada N (2017) Host-microbial cross-talk in inflammatory bowel disease. Immune Netw 17:1-12. https:// doi.org/10.4110/in.2017.17.1.1

36. Hall AB, Yassour M, Sauk J et al (2017) A novel Ruminococcus gnavus clade enriched in inflammatory bowel disease patients. Genome Med 9:103. https://doi.org/10.1186/s13073-017-0490-5

37. Prosberg M, Bendtsen F, Vind I et al (2016) The association between the gut microbiota and the inflammatory bowel disease activity: a systematic review and meta-analysis. Scand J Gastroenterol 51:1407-1415. https://doi.org/10.1080/00365521.2016. 1216587

38. Sankarasubramanian J, Ahmad R, Avuthu N et al (2020) Gut microbiota and metabolic specificity in ulcerative colitis and Crohn's disease. Front Med. https://doi.org/10.3389/fmed.2020. 606298
39. Willing BP, Dicksved J, Halfvarson J et al (2010) A pyrosequencing study in twins shows that gastrointestinal microbial profiles vary with inflammatory bowel disease phenotypes. Gastroenterology 139:1844-1854.e1. https://doi.org/10.1053/j.gastro.2010. 08.049

40. Gibson PR (2017) Use of the low-FODMAP diet in inflammatory bowel disease. J Gastroenterol Hepatol 32:40-42. https://doi.org/ 10.1111/jgh. 13695

41. Hsieh M-S, Hsu W-H, Wang J-W et al (2019) Nutritional and dietary strategy in the clinical care of inflammatory bowel disease. J Formos Med Assoc S0929-S6646(19):30468-30471. https://doi.org/10.1016/j.jfma.2019.09.005

42. Raghu Subramanian C, Triadafilopoulos G (2016) Care of inflammatory bowel disease patients in remission. Gastroenterol Rep 4:261-271. https://doi.org/10.1093/gastro/gow032

43. Starz E, Wzorek K, Folwarski M et al (2021) The modification of the gut microbiota via selected specific diets in patients with Crohn's disease. Nutrients. https://doi.org/10.3390/nu13072125

44. Kakodkar S, Farooqui AJ, Mikolaitis SL, Mutlu EA (2015) The specific carbohydrate diet for inflammatory bowel disease: a case series. J Acad Nutr Diet 115:1226-1232. https://doi.org/ 10.1016/j.jand.2015.04.016

45. Adamji M, Day AS (2019) An overview of the role of exclusive enteral nutrition for complicated Crohn's disease. Intest Res 17:171-176. https://doi.org/10.5217/ir.2018.00079

46. Niland B, Cash BD (2018) Health benefits and adverse effects of a gluten-free diet in non-celiac disease patients. Gastroenterol Hepatol (N Y) 14:82-91

47. Olendzki BC, Silverstein TD, Persuitte GM et al (2014) An anti-inflammatory diet as treatment for inflammatory bowel disease: a case series report. Nutr J 13:5. https://doi.org/10.1186/ 1475-2891-13-5

48. Chicco F, Magrì S, Cingolani A et al (2021) Multidimensional impact of Mediterranean diet on IBD patients. Inflamm Bowel Dis 27:1-9. https://doi.org/10.1093/ibd/izaa097

49. Willett WC, Sacks F, Trichopoulou A et al (1995) Mediterranean diet pyramid: a cultural model for healthy eating. Am J Clin Nutr 61:1402S-1406S. https://doi.org/10.1093/ajen/61.6.1402S

50. Marlow G, Ellett S, Ferguson IR et al (2013) Transcriptomics to study the effect of a Mediterranean-inspired diet on inflammation in Crohn's disease patients. Hum Genomics 7:24. https://doi.org/ 10.1186/1479-7364-7-24

51. Weber AT, Shah ND, Sauk J, Limketkai BN (2019) Popular diet trends for inflammatory bowel diseases: claims and evidence. Curr Treat Options Gastroenterol 17:564-576. https://doi.org/ 10.1007/s11938-019-00248-Z

52. Gibson PR, Shepherd SJ (2005) Personal view: food for thought - western lifestyle and susceptibility to Crohn's disease. The FODMAP hypothesis. Aliment Pharmacol Ther 21:1399-1409. https://doi.org/10.1111/j.1365-2036.2005.02506.x

53. Vandeputte D, Joossens M (2020) Effects of low and high FODMAP diets on human gastrointestinal microbiota composition in adults with intestinal diseases: a systematic review. Microorganisms 8:1638. https://doi.org/10.3390/microorganisms8111638

54. Grammatikopoulou MG, Goulis DG, Gkiouras K et al (2020) Low FODMAP diet for functional gastrointestinal symptoms in quiescent inflammatory bowel disease: a systematic review of randomized controlled trials. Nutrients 12:3648. https://doi.org/ $10.3390 /$ nu 12123648

55. Gibson PR, Shepherd SJ (2005) Personal view: food for thoughtwestern lifestyle and susceptibility to Crohn's disease. The FODMAP hypothesis. Aliment Pharmacol Ther 21:1399-1409. https://doi.org/10.1111/j.1365-2036.2005.02506.x

56. Chelakkot C, Ghim J, Ryu SH (2018) Mechanisms regulating intestinal barrier integrity and its pathological implications. Exp Mol Med 50:1-9. https://doi.org/10.1038/s12276-018-0126-х 
57. Cox SR, Prince AC, Myers CE et al (2017) Fermentable carbohydrates [FODMAPs] exacerbate functional gastrointestinal symptoms in patients with inflammatory bowel disease: a randomised, double-blind, placebo-controlled, cross-over, re-challenge trial. J Crohns Colitis 11:1420-1429. https://doi.org/10.1093/ecco-jcc/ jjx073

58. Nelson MDLC (2000) Lehninger principles of biochemistry, 3rd edn. Worth Publishers, New York

59. Ferraris RP, Choe J-Y, Patel CR (2018) Intestinal absorption of fructose. Annu Rev Nutr 38:41-67. https://doi.org/10.1146/annur ev-nutr-082117-051707

60. Beisner J, Gonzalez-Granda A, Basrai M et al (2020) Fructoseinduced intestinal microbiota shift following two types of shortterm high-fructose dietary phases. Nutrients 12:3444. https://doi. org/10.3390/nu12113444

61. Pryde SE, Duncan SH, Hold GL et al (2002) The microbiology of butyrate formation in the human colon. FEMS Microbiol Lett 217:133-139. https://doi.org/10.1111/j.1574-6968.2002.tb114 67. $\mathrm{x}$

62. Schirmer M, Smeekens SP, Vlamakis H et al (2016) Linking the human gut microbiome to inflammatory cytokine production capacity. Cell 167:1125-1136.e8. https://doi.org/10.1016/j.cell. 2016.10.020

63. Di Costanzo M, Berni Canani R (2018) Lactose intolerance: common misunderstandings. Ann Nutr Metab 73(suppl 4):30-37. https://doi.org/10.1159/000493669

64. Brandao Gois MF, Sinha T, Spreckels JE et al (2021) Role of the gut microbiome in mediating lactose intolerance symptoms. Gut. https://doi.org/10.1136/gutjnl-2020-323911

65. Kato K, Ishida S, Tanaka M et al (2018) Association between functional lactase variants and a high abundance of Bifidobacterium in the gut of healthy Japanese people. PLoS ONE 13:e0206189. https://doi.org/10.1371/journal.pone.0206189

66. Marcason W (2012) What Is the FODMAP diet? J Acad Nutr Diet 112:1696. https://doi.org/10.1016/j.jand.2012.08.005

67. Roberfroid MB (2005) Introducing inulin-type fructans. Br J Nutr 93:S13-S25. https://doi.org/10.1079/BJN20041350

68. Wilson B, Whelan K (2017) Prebiotic inulin-type fructans and galacto-oligosaccharides: definition, specificity, function, and application in gastrointestinal disorders. J Gastroenterol Hepatol 32:64-68. https://doi.org/10.1111/jgh.13700

69. Gibson GR, Roberfroid MB (1995) Dietary modulation of the human colonic microbiota: introducing the concept of prebiotics. J Nutr 125:1401-1412. https://doi.org/10.1093/jn/125.6.1401

70. Krumbeck JA, Rasmussen HE, Hutkins RW et al (2018) Probiotic Bifidobacterium strains and galactooligosaccharides improve intestinal barrier function in obese adults but show no synergism when used together as synbiotics. Microbiome 6:121. https://doi. org/10.1186/s40168-018-0494-4

71. Liu F, Li P, Chen M et al (2017) Fructooligosaccharide (FOS) and galactooligosaccharide (GOS) increase bifidobacterium but reduce butyrate producing bacteria with adverse glycemic metabolism in healthy young population. Sci Rep 7:11789. https://doi. org/10.1038/s41598-017-10722-2

72. Tandon D, Haque MM, Gote M et al (2019) A prospective randomized, double-blind, placebo-controlled, dose-response relationship study to investigate efficacy of fructo-oligosaccharides (FOS) on human gut microflora. Sci Rep 9:5473. https://doi.org/ 10.1038/s41598-019-41837-3

73. Birkeland E, Gharagozlian S, Birkeland KI et al (2020) Prebiotic effect of inulin-type fructans on faecal microbiota and short-chain fatty acids in type 2 diabetes: a randomised controlled trial. Eur J Nutr 59:3325-3338. https://doi.org/10.1007/ s00394-020-02282-5

74. Iraporda C, Errea A, Romanin DE et al (2015) Lactate and short chain fatty acids produced by microbial fermentation downregulate proinflammatory responses in intestinal epithelial cells and myeloid cells. Immunobiology 220:1161-1169. https://doi.org/10.1016/j.imbio.2015.06.004

75. Schouler C, Taki A, Chouikha I et al (2020) A genomic island of an extraintestinal pathogenic Escherichia coli strain enables the metabolism of fructooligosaccharides, which improves intestinal colonization. J Bacteriol 191:388-393. https://doi. org/10.1128/JB.01052-08

76. Mao B, Li D, Zhao J et al (2015) In vitro fermentation of fructooligosaccharides with human gut bacteria. Food Funct 6:947-954. https://doi.org/10.1039/C4FO01082E

77. Livesey G (1992) The energy values of dietary fibre and sugar alcohols for man. Nutr Res Rev 5:61-84. https://doi.org/10. 1079/NRR19920007

78. Lenhart A, Chey WD (2017) A systematic review of the effects of polyols on gastrointestinal health and irritable bowel syndrome. Adv Nutr 8:587-596. https://doi.org/10.3945/an.117. 015560

79. Grembecka M (2015) Sugar alcohols - their role in the modern world of sweeteners: a review. Eur Food Res Technol 241:1-14. https://doi.org/10.1007/s00217-015-2437-7

80. Varney J, Barrett J, Scarlata K et al (2017) FODMAPs: food composition, defining cutoff values and international application. J Gastroenterol Hepatol 32:53-61. https://doi.org/10.1111/jgh. 13698

81. Yao CK, Tan H-L, van Langenberg DR et al (2014) Dietary sorbitol and mannitol: food content and distinct absorption patterns between healthy individuals and patients with irritable bowel syndrome. J Hum Nutr Diet 27:263-275. https://doi.org/10.1111/ jhn. 12144

82. Sato T, Kusuhara S, Yokoi W et al (2017) Prebiotic potential of L-sorbose and xylitol in promoting the growth and metabolic activity of specific butyrate-producing bacteria in human fecal culture. FEMS Microbiol Ecol. https://doi.org/10.1093/femsec/ fiw 227

83. Chang Y-C, Ching Y-H, Chiu C-C et al (2017) TLR2 and interleukin-10 are involved in Bacteroides fragilis-mediated prevention of DSS-induced colitis in gnotobiotic mice. PLoS ONE 12:e0180025

84. Lo Presti A, Zorzi F, Del Chierico F et al (2019) Fecal and mucosal microbiota profiling in irritable bowel syndrome and inflammatory bowel disease. Front Microbiol 10:1655. https:// doi.org/10.3389/fmicb.2019.01655

85. Mäkeläinen HS, Mäkivuokko HA, Salminen SJ et al (2007) The effects of polydextrose and xylitol on microbial community and activity in a 4-stage colon simulator. J Food Sci 72:M153-M159. https://doi.org/10.1111/j.1750-3841.2007.00350.x

86. Gostner A, Blaut M, Schäffer V et al (2006) Effect of isomalt consumption on faecal microflora and colonic metabolism in healthy volunteers. Br J Nutr 95:40-50. https://doi.org/10.1079/ BJN20051589

87. Grimble GK, Patil DH, Silk DB (1988) Assimilation of lactitol, an "unabsorbed" disaccharide in the normal human colon. Gut 29:1666-1671. https://doi.org/10.1136/gut.29.12.1666

88. Finney M, Smullen J, Foster HA et al (2007) Effects of low doses of lactitol on faecal microflora, $\mathrm{pH}$, short chain fatty acids and gastrointestinal symptomology. Eur J Nutr 46:307. https://doi. org/10.1007/s00394-007-0666-7

89. Gearry RB, Irving PM, Barrett JS et al (2009) Reduction of dietary poorly absorbed short-chain carbohydrates (FODMAPs) improves abdominal symptoms in patients with inflammatory bowel disease-a pilot study. J Crohn's Colitis 3:8-14. https:// doi.org/10.1016/j.crohns.2008.09.004

90. Prince AC, Myers CE, Joyce T et al (2016) Fermentable carbohydrate restriction (low FODMAP diet) in clinical practice improves functional gastrointestinal symptoms in patients with 
inflammatory bowel disease. Inflamm Bowel Dis 22:1129-1136. https://doi.org/10.1097/MIB.0000000000000708

91. Cohen AB, Lee D, Long MD et al (2013) Dietary patterns and self-reported associations of diet with symptoms of inflammatory bowel disease. Dig Dis Sci 58:1322-1328. https://doi.org/ 10.1007/s10620-012-2373-3

92. Barbalho SM, de Goulart R, de Aranão ALC, de Oliveira PGC (2018) Inflammatory bowel diseases and fermentable oligosaccharides, disaccharides, monosaccharides, and polyols: an overview. J Med Food 21:633-640. https://doi.org/10.1089/jmf.2017. 0120

93. Gibson PR (2011) Food intolerance in functional bowel disorders. J Gastroenterol Hepatol 26(Suppl 3):128-131. https://doi. org/10.1111/j.1440-1746.2011.06650.x

94. Halmos EP (2016) A low FODMAP diet in patients with Crohn's disease. J Gastroenterol Hepatol 31(Suppl 1):14-15. https://doi. org/10.1111/jgh.13349

95. Gibson PR, Shepherd SJ (2010) Evidence-based dietary management of functional gastrointestinal symptoms: the FODMAP approach. J Gastroenterol Hepatol 25:252-258. https://doi.org/ 10.1111/j.1440-1746.2009.06149.x

96. Pedersen N, Ankersen DV, Felding M et al (2017) Low-FODMAP diet reduces irritable bowel symptoms in patients with inflammatory bowel disease. World J Gastroenterol 23:33563366. https://doi.org/10.3748/wjg.v23.i18.3356

97. Bodini G, Zanella C, Crespi M et al (2019) A randomized, 6-week trial of a low FODMAP diet in patients with inflammatory bowel disease. Nutrition 67-68:110542. https://doi.org/10. 1016/j.nut.2019.06.023

98. Maagaard L, Ankersen DV, Végh Z et al (2016) Follow-up of patients with functional bowel symptoms treated with a low FODMAP diet. World J Gastroenterol 22:4009-4019. https:// doi.org/10.3748/wjg.v22.i15.4009

99. Croagh C, Shepherd SJ, Berryman M et al (2007) Pilot study on the effect of reducing dietary FODMAP intake on bowel function in patients without a colon. Inflamm Bowel Dis 13:1522-1528. https://doi.org/10.1002/ibd.20249

100. Staudacher HM, Irving PM, Lomer MCE, Whelan K (2014) Mechanisms and efficacy of dietary FODMAP restriction in IBS. Nat Rev Gastroenterol Hepatol 11:256-266. https://doi.org/10. 1038/nrgastro.2013.259

101. Zhan Y, Zhan Y-A, Dai S-X (2018) Is a low FODMAP diet beneficial for patients with inflammatory bowel disease? A metaanalysis and systematic review. Clin Nutr 37:123-129. https:// doi.org/10.1016/j.clnu.2017.05.019

102. Damas OM, Garces L, Abreu MT (2019) Diet as adjunctive treatment for inflammatory bowel disease: review and update of the latest literature. Curr Treat Options Gastroenterol 17:313-325. https://doi.org/10.1007/s11938-019-00231-8

103. Kakodkar S, Mutlu EA (2017) Diet as a therapeutic option for adult inflammatory bowel disease. Gastroenterol Clin North Am 46:745-767. https://doi.org/10.1016/j.gtc.2017.08.016

104. Stein AC, Gaetano JN, Jacobs J et al (2016) Northern latitude but not season is associated with increased rates of hospitalizations related to inflammatory bowel disease: results of a multi-year analysis of a national cohort. PLoS ONE 11:e161523. https:// doi.org/10.1371/journal.pone.0161523
105. Bishehsari F, Voigt RM, Keshavarzian A (2020) Circadian rhythms and the gut microbiota: from the metabolic syndrome to cancer. Nat Rev Endocrinol 16:731-739. https://doi.org/10. 1038/s41574-020-00427-4

106. Siva S, Rubin DT, Gulotta G et al (2017) Zinc deficiency is associated with poor clinical outcomes in patients with inflammatory bowel disease. Inflamm Bowel Dis 23:152-157. https://doi.org/ 10.1097/MIB.0000000000000989

107. Gröber U, Reichrath J, Holick MF (2015) Live longer with vitamin D? Nutrients 7:1871-1880. https://doi.org/10.3390/nu703 1871

108. EFSA Panel on Dietetic Products, Nutrition and Allergies (2014) Scientific opinion on dietary reference values for zinc. EFSA J 12:3844. https://doi.org/10.2903/j.efsa.2014.3844

109. Cox SR, Lindsay JO, Fromentin S et al (2020) Effects of low FODMAP diet on symptoms, fecal microbiome, and markers of inflammation in patients with quiescent inflammatory bowel disease in a randomized trial. Gastroenterology 158:176-188.e7. https://doi.org/10.1053/j.gastro.2019.09.024

110. Marsh A, Eslick EM, Eslick GD (2016) Does a diet low in FODMAPs reduce symptoms associated with functional gastrointestinal disorders? A comprehensive systematic review and meta-analysis. Eur J Nutr 55:897-906. https://doi.org/10.1007/ s00394-015-0922-1

111. Gu P, Feagins LA (2020) Dining with inflammatory bowel disease: a review of the literature on diet in the pathogenesis and management of IBD. Inflamm Bowel Dis 26:181-191. https:// doi.org/10.1093/ibd/izz268

112. Andersen V, Chan S, Luben R et al (2018) Fibre intake and the development of inflammatory bowel disease: a European prospective multi-centre cohort study (EPIC-IBD). J Crohn's Colitis. https://doi.org/10.1093/ecco-jcc/jjx136

113. Halmos EP, Christophersen CT, Bird AR et al (2016) Consistent prebiotic effect on gut microbiota with altered FODMAP intake in patients with Crohn's disease: a randomised, controlled crossover trial of well-defined diets. Clin Transl Gastroenterol 7:164. https://doi.org/10.1038/ctg.2016.22

114. Nishida A, Inoue R, Inatomi O et al (2018) Gut microbiota in the pathogenesis of inflammatory bowel disease. Clin J Gastroenterol 11:1-10. https://doi.org/10.1007/s12328-017-0813-5

115. Sokol H, Pigneur B, Watterlot L et al (2008) Faecalibacterium prausnitzii is an anti-inflammatory commensal bacterium identified by gut microbiota analysis of Crohn disease patients. Proc Natl Acad Sci 105:16731-16736. https://doi.org/10.1073/pnas. 0804812105

116. Yao CK, Muir JG, Gibson PR (2016) Review article: insights into colonic protein fermentation, its modulation and potential health implications. Aliment Pharmacol Ther 43:181-196. https://doi. org/10.1111/apt.13456

117. Diether NE, Willing BP (2019) Microbial fermentation of dietary protein: an important factor in diet $^{-}$microbe ${ }^{-}$host interaction. Microorganisms 7:19. https://doi.org/10.3390/microorganisms7 010019

118. Hill P, Muir JG, Gibson PR (2017) Controversies and recent developments of the low-FODMAP diet. Gastroenterol Hepatol $13: 36-45$ 